\title{
BuST: Budget Sharing Token Protocol for Hard Real-Time Communication
}

\author{
Gianluca Franchino \\ University of Pavia, Italy \\ gianluca.franchino@unipv.it
}

\author{
Giorgio C. Buttazzo \\ Scuola Superiore S. Anna, Italy \\ giorgio.buttazzo@sssup.it
}

\author{
Tullio Facchinetti \\ University of Pavia, Italy \\ tullio.facchinetti@unipv.it
}

\begin{abstract}
Timed-token networks, such as FDDI, support both synchronous real-time traffic and non real-time traffic (asynchronous messages). The medium access scheme of FDDI guarantees up to one half of the total network bandwidth for synchronous communication. Further enhancements, such as FDDI-M, improve the bandwidth dedicated to real-time messages. However, the ability of timed-token protocols to guarantee synchronous message deadlines highly depends on specific Synchronous Budget Allocation (SBA) schemes.

This paper introduces BuST, the Budget Sharing Token protocol which improves the management of periodic real-time traffic, while guaranteeing a minimum throughput for non real-time messages, with respect to existing techniques. We evaluate the performance of BuST, in comparison with FDDI and FDDI-M, considering a Synchronous Budget Allocation (SBA) scheme proposed in the literature, using the Worst-Case Achievable Utilization (WCAU) as performance metrics. We demonstrate that the performance achieved by BuST is better or, in a few cases, equal to FDDI and FDDI-M.
\end{abstract}

\section{Introduction}

The complexity of modern real-time applications like train, car, and airplane control requires the employment of several distributed, interconnected and cooperating onboard embedded systems. The correct behavior of such systems depends on the timely execution of tasks that are often executed on different nodes and need to share global information to work properly.

One of the most effective solutions for the real-time communication among distributed devices is the timed token approach. Timed token protocols are token-passing disciplines in which each node receives a guaranteed share of the network bandwidth. A token travels between nodes in a circular fashion and each node can transmit only when it possesses the token; this guarantees a collisionfree medium access. Timed token protocols [8, 12, 17] are used in some networks like fiber distributed data interface (FDDI), survivable adaptable fiber optic embedded network (SAFENET) [11], modified fiber distributed data interface (FDDI-M), and in PROFIBUS [16] which is one of the most popular field bus technology. In particular, we analyze the properties of the proposed approach in comparison with the MAC protocol of FDDI and FDDI-M networks.

Token passing protocols have some good features that make them attractive for real applications. For instance, they do not need clocks synchronization among the nodes. Moreover, because of the token passing function, they have an implicit bandwidth reclaiming mechanism such that the bandwidth not used by a node can be exploited by the following ones.

There exist several applications based on timed token protocols. For instance, in [13] the authors show the usage of the protocol to guarantee a minimum $Q o S$ for the transmission of MPEG video. Bussiere et al [3], describe a submarine application using a distributed architecture, where hard real-time requirements are defined both for the computational activities and the data communication. The application adopts several standards, as ADA for the language, POSIX 1003.1 for the operating system, and SAFENET as communication technology. A wireless version of the timed token protocol has been developed in [6], as communication infrastructure for the coordination of a mobile robots team.

In real-time communication systems, messages can be grouped in two classes: synchronous and asynchronous. The former class is primarily used for real-time messages with periodic arrival pattern, whereas the latter is used for non real-time aperiodic messages with unknown arrival time.

In timed token approaches, an important parameter is the so-called Target Token Rotation Time (TTRT), which represents the expected time needed by the token to complete an entire round-trip of the network. Each node $i$ has an associated time budget $H_{i}$; whenever a node receives the token, it can transmit its synchronous messages for a time no greater than $H_{i}$. It can then transmit its asynchronous messages if the time elapsed since the previous token departure from the same node is less than the value of TTRT, that is, only if the token arrives earlier than expected. To assign the budget $H_{i}$ to each node, several allocation rules have been proposed during the past years. These rules are named Synchronous Budget Allocation (SBA) schemes.

\subsection{Performance metrics}

For evaluating and comparing the performance of different SBA schemes in a timed token network, several metrics have been proposed. One of the most widely adopted metrics is the Worst Case Achievable Utilization (WCAU) [9, 18]. The WCAU of an SBA scheme represents the largest utilization $\left(U^{*}\right)$ of the network such that, for any real-time message set whose total network utilization is $U \leq U^{*}$, the SBA scheme can guarantee the timeliness of each single real-time message. For a formal definition of the network utilization $U$, see Section 2 .

The WCAU test is useful to guarantee the feasibility of a real-time message set when only an estimation of the amount of real-time traffic in known (i.e., the maximum time required to send a message) without requiring a detailed characterization of each single real-time message. 


\subsection{Contributions and summary}

This paper describes the BuST (Budget Sharing Token) protocol, which improves the characteristics of two existing timed-token based techniques: FDDI and FDDI-M. The BuST protocol differs from FDDI and FDDI-M in how each node exploits the bandwidth saved during the round trip of the token, if any, to deliver non real-time (asynchronous) traffic. The transmission of non real-time traffic occurs within the spare budget unused by real-time (synchronous) traffic, even when the token is not early. In other words, the budget of a node is shared between synchronous and asynchronous traffic. Such a difference will be better explained in the next sections.

This paper compares the BuST timing properties with those of FDDI and FDDI-M using the Worst-Case Achievable Utilization (WCAU), which is evaluated under the Proportional Allocation (PA) scheme, which is analyzed in Section 4.

Theoretical results show that, with respect to FDDI and FDDI-M, for the PA scheme BuST has a higher WCAU.

This paper also derives some new results about the FDDI-M protocol. In particular, the analysis shows that the maximum bandwidth dedicated to non real-time messages by FDDI-M is zero for the Normalized Proportional Allocation (NPA) scheme, whereas it depends on the channel utilization when using the PA scheme.

The rest of the paper is organized as follows: Section 2 introduces the adopted communication model; Section 3 describes the classical timed token protocols (FDDI, FDDI-M) and presents the features of the BuST protocol in detail; Section 4 introduces the budget allocation schemes considered in this paper; Section 5 provides some theoretical results as the WCAUs. Section 6 shows some simulation results and, finally, Section 7 states our conclusions and future work.

\section{Communication model}

The communication system is composed by a set of $n$ communicating nodes. Each node $i$ is associated with a synchronous message stream $S_{i}$, which is described by three parameters $\left(C_{i}, T_{i}, D_{i}\right)$, where:

- $C_{i}$ is the maximum amount of time required to transmit a message in the stream. This includes the time required to transmit both the payload data and the message headers.

- $T_{i}$ is the interarrival period between consecutive messages in stream $S_{i}$. If the first message of node $i$ is put in the transmission queue at time $t_{i, 1}$, then the $j$ th message in stream $S_{i}$ will arrive at node $i$ at time $t_{i, j}=t_{i, 1}+(j-1) T_{i}$, where $j \geq 1$.

- $D_{i}$ is the relative deadline associated with messages in stream $S_{i}$, that is, the maximum amount of time that can elapse between a message arrival and the completion of its transmission. Thus, the transmission of the $j$-th message in stream $S_{i}$ that arrives at $t_{i, j}$ must be completed not later than $d_{i}=t_{i, j}+D_{i}$, which is the message's absolute deadline.

Without loss of generality, we assume only one synchronous stream per node. In fact, as proved in [1], a timed token network with more than one stream per node can be transformed into a logically equivalent network with one synchronous stream per (logical) node.

Notice that, in order to guarantee the deadlines of asynchronous real-time messages, if any, we can use the stream model described above and assign a budget for this kind of messages, as proposed in [12]. $S_{i}$ is

The channel utilization of each message in the stream

$$
U_{i}=\frac{C_{i}}{\min \left(T_{i}, D_{i}\right)} .
$$

The total effective channel utilization, $U$, of a periodic message set is then

$$
U=\sum_{i=1}^{n} U_{i}
$$

which measures the total channel bandwidth required by the whole periodic message set.

The parameters described above are crucial for guaranteeing periodic messages deadlines. Before discussing how to select the communication parameters, we introduce the following definitions:

Definition $1 \tau$ is the time needed to transmit the token between nodes, including the overhead introduced by the protocol.

Any choice of the communication parameters must satisfy the following two constraints:

Definition 2 (Protocol Constraint) The total bandwidth allocated to the nodes must be less than the available network bandwidth, that is,

$$
\frac{\sum_{i=1}^{n} H_{i}}{T T R T} \leq 1-\frac{\tau}{T T R T} .
$$

The Protocol Constraint is necessary to ensure a stable operation of the timed-token protocol.

Definition 3 (Deadline Constraint) If $s_{i, j}$ is the time at which the transmission of the $j$-th message in stream $S_{i}$ is completed, the deadline constraint requires that for $i=$ $1, \ldots, n$ and $j=1,2, \ldots$,

$$
s_{i, j} \leq t_{i, j}+D_{i}
$$

where $t_{i, j}$ is the message arrival time and $D_{i}$ is its relative deadline.

The Deadline Constraint ensures that every periodic message is transmitted before its absolute deadline. Note that in the above inequality, while $t_{i, j}$ and $D_{i}$ are defined by the application, $s_{i, j}$ depends on the synchronous bandwidth allocation and on the TTRT value.

\section{FDDI, FDDI-M and BuST protocols}

This section presents a brief description of the FDDI and FDDI-M protocols and, after discussing the drawbacks of these approaches, it introduces the BuST protocol and its features.

\subsection{The FDDI MAC protocol}

In FDDI networks, nodes access to a shared channel under the following rules:

1. During the ring initialization phase, each node $i$ declares a TTRT value equal to one half of the deadline $D_{i}$ of its synchronous message stream. The minimum declared value is selected as the ring's TTRT. Each node $i$ is then assigned a slice $H_{i}$ of the TTRT to transmit its synchronous traffic. 
2. Each node has two timers, the token holding timer $(T H T)$ and the token-rotation-timer (TRT). The TRT counter always increases, whereas the THT only increases when the node is delivering asynchronous traffic. When TRT reaches TTRT, it is reset to 0 and the token is signed as "late" by incrementing the node's late count $L_{c}$ by one. To initialize the timers and $L_{c}$, no messages are sent during the first token rotation after the ring initialization.

3. Only the node holding the token can transmit messages. Transmission is controlled by the timers, but an in-progress transmission of a single packet is not interrupted until its completion. When node $i$ gets the token, it performs the following operations:

(a) If $L_{c}>0$, it sets $L_{c}=L_{c}-1$ and $T H T=T T R T$. Otherwise, THT $=T R T$ and $T R T=0$.

(b) If node $i$ has synchronous packets, it transmits them for a time no greater than $H_{i}$.

(c) If node $i$ has asynchronous packets, it transmits them until THT counts up to TTRT, or until all the asynchronous traffic is sent, which ever comes first.

(d) Node $i$ passes the token to station $(i+1)$ $\bmod (n+1)$.

\subsubsection{Drawbacks of FDDI}

Johnson and Sevciks [14] showed that the average token rotation time, i.e. the average interval between two consecutive visits of the token at the same node, does not exceed TTRT and the worst-case token rotation time does not exceed $2 T T R T$. This last result suggests that, if $D$ is the minimum deadline of the synchronous messages, to ensure the satisfaction of $D, T T R T$ has to be no greater than $D / 2$. This explains the choice of the TTRT during the initialization phase. Consequently, due to the protocol constraint, the total synchronous bandwidth must be no greater than $\left(\frac{1}{2}-\frac{\tau}{D}\right)$. Since the actual available bandwidth is $\left(1-\frac{\tau}{D}\right)$, the maximum bandwidth available for synchronous messages is half of the total bandwidth. This is the main drawback of the FDDI MAC protocol.

\subsection{The FDDI-M MAC protocol}

To eliminate the FDDI drawback illustrated in Section 3.1.1, Shin and Zheng [15] proposed a modification of FDDI, called FDDI-M, where the maximum time allocated to asynchronous messages is equal to TTRT $\sum_{i=1}^{n} H_{i}-\tau$. Note that, with FDDI, the maximum time allocated to asynchronous messages is equal to TTRT $\tau$. Chan et al. [4] proved that for FDDI-M, the maximum amount of time between two consecutive token arrivals at a given node is TTRT. From the protocol constraint, the total synchronous bandwidth must be no greater than $\left(1-\frac{\tau}{D}\right)$, which is the maximum available bandwidth.

To fully exploit the bandwidth for real-time traffic, FDDI-M limits the worst-case token rotation time to TTRT. Therefore, the TTRT can be equal to the minimum message deadline $D$, instead of $D / 2$.

The token rotation time is composed by three components: the time for the synchronous packets, the time for asynchronous packets, and the protocol overhead $\tau$. During the token rotation, the time used for the synchronous traffic is bounded by $T_{S}=\sum_{i=1}^{n} H_{i}$. It follows that, in order to limit the maximum token rotation time to TTRT, the transmission of asynchronous traffic must be bounded by $T_{A}=T T R T-T_{S}-\tau$. In FDDI-M, this is achieved by means of the following modification to FDDI:

1. Use a new target token rotation time $T T R T_{n}=$ TTRT $-T_{S}$, instead of the TTRT;

2. Stop the counting of a node's token rotation timer $(T R T)$ when a synchronous message is being delivered by the node.

Adding these modifications to the FDDI MAC standard, the FDDI-M MAC protocol works as follows:

1. During the ring initialization phase, each node $i$ declares a TTRT value equal to the deadline $D_{i}$ of its synchronous message stream. The minimum declared value is selected as the ring's TTRT. Each node $i$ is then assigned a slice $H_{i}$ of the TTRT to transmit its synchronous traffic, and then sets $\operatorname{TTRT}_{n}=\operatorname{TTRT}-\sum_{i=1}^{n} H_{i}$.

2. Each node has two timers, the token holding timer $(T H T)$ and the token-rotation-timer $(T R T)$. The $T R T$ increases only when the node is not transmitting synchronous traffic, whereas the THT increases only when the node is delivering asynchronous traffic. To initialize the timers, no messages are sent during the first token rotation after the ring initialization.

3. Only the node holding the token can transmit messages. The transmission is controlled by the timers, but an in-progress transmission of a single packet is not interrupted until its completion. When node $i$ gets the token, it performs the following operations:

(a) $T H T=T R T$ and $T R T=0$.

(b) If node $i$ has synchronous packets, it transmit them for a time up to $H_{i}$, or until all the synchronous traffic is sent, whichever comes first.

(c) If node $i$ has asynchronous packets, it transmits them until THT counts up to $T T R T_{n}$, or until all the asynchronous traffic is sent, whichever comes first.

(d) Node $i$ passes the token to station $(i+1)$ $\bmod (n+1)$.

\subsubsection{Drawbacks of FDDI-M}

FDDI-M guarantees that the worst-case token rotation time does not exceed TTRT. This important enhancement allows allocating all the bandwidth to real-time streams. Moreover, the protocol overhead of FDDI-M results in a bandwidth waste equal to $\frac{\tau}{T T R T}=\frac{\tau}{D}$, while for FDDI the waste is double, i.e., equal to $\frac{2 \tau}{D}$.

Despite the nice properties of FDDI-M illustrated above, its main problem is that, in some cases, a node cannot deliver any asynchronous traffic. To see this, we anticipate the introduction of the synchronous allocation schemes (SBA), that will be considered later in the paper. Table 1 shows the SBA schemes, i.e. the rules to assign the budgets $H_{i}$ (see Section 4 for further details). Recalling that the maximum time a node can use to deliver non real-time traffic is $T_{A}=T T R T-\sum_{i=1}^{n} H_{i}-\tau$, it can be observed that:

- For the PA scheme, $T_{A}=$ TTRT $\sum_{i=1}^{n} U_{i}(T T R T-\tau)-\tau=T T R T-U(T T R T-$ $\tau)-\tau=(1-U)(T T R T-\tau)$.

- For the NPA scheme, $T_{A}=$ TTRT $\sum_{i=1}^{n} \frac{U_{i}}{U}(T T R T-\tau)-\tau=T T R T-(T T R T-$ $\tau)-\tau=0$. 
This means that FDDI-M is not able to deliver non realtime traffic under the NPA scheme, while $T_{A}$ depends on $U$ using the PA scheme.

\subsection{The BuST MAC protocol}

The goal of the BuST protocol is to improve the original timed-token rules in order to avoid the problems of FDDI and FDDI-M highlighted in the previous sections. In particular, the worst-case token rotation time is limited so that it cannot exceed the TTRT, which improves FDDI and allow a node to deliver non real-time traffic in those cases where FDDI-M fails.

Like in the traditional timed token policy, the BuST protocol assigns each node a time budget $H_{i}$ for transmitting its real-time traffic. When a node receives the token, it can transmit the associated real-time traffic for a time no greater than the corresponding budget. The main difference with respect to FDDI and FDDI-M concerns the non real-time message service. Using FDDI, when the token arrives early, the node can transmit asynchronous traffic for a time no greater than $T_{A}=T T R T-\tau-T_{L R T}$, where $T_{L R T}$ is the time spent in the last round-trip of the token. Using FDDI-M a node does the same but with $T_{A}=T T R T-\sum_{i=1}^{n} H_{i}-\tau$. In BuST, a node can deliver non real-time traffic each time it gets the token, early or not, using the spare budget left by real-time messages. If $H_{i}^{\text {cons }}$ is the budget consumed by node $i$ to deliver synchronous traffic, then it can send asynchronous traffic for a time no greater than $T_{A_{i}}=H_{i}-H_{i}^{\text {cons }}$, even if the token is not early. Observe that, FDDI and FDDI-M can deliver asynchronous traffic only when the token is early, that is, when $T_{L R T}<T T R T-\tau$.

The specific rules defining the BuST protocol are shown below.

1. During the ring initialization phase, each node $i$ declares a TTRT value equal to the deadline $D_{i}$ of its synchronous message stream. The minimum declared value is selected as the ring's TTRT. Each node $i$ is then assigned a slice $H_{i}$ of the TTRT to transmit its synchronous traffic.

2. Each node has one timer, the token holding-rotation timer $(T H R T)$. The THRT counter always increases. To initialize timers, no messages are sent during the first token rotation.

3. Only the node having the token can transmit messages. The transmission is controlled by the timer, but an in-progress transmission of a single packet is not interrupted until its completion. When node $i$ gets the token, it performs the following operations:

(a) It sets $T H R T=0$.

(b) If node $i$ has synchronous packets, it transmits them until THRT counts up to $H_{i}$, or until all the synchronous traffic is sent, whichever comes first.

(c) If node $i$ has asynchronous packets, it transmits them until THRT counts up to $H_{i}$, or until all the asynchronous traffic is sent, which ever comes first.

(d) If a synchronous message becomes ready during the transmission of asynchronous packets, and THRT $<H_{i}$ the transmission is stopped, and the node starts delivering the real-time traffic until THRT counts up to $H_{i}$, or until all the synchronous traffic is sent, whichever comes first. (e) If node $i$ completes the transmission of the synchronous traffic without consuming entirely its budget, i.e. THRT $<H_{i}$, it starts transmitting its asynchronous traffic, if any, until THRT counts up to $H_{i}$, or until all the asynchronous traffic is sent, which ever comes first. Note that, in this case, the transmission is not stopped even if a real-time message becomes ready.

(f) Node $i$ passes the token to station $(i+1)$ $\bmod (n+1)$.

In BuST, node $i$ can use its budget $H_{i}$ for delivering both real-time and non real-time messages. Therefore, the worst-case token rotation time cannot exceed TTRT. With respect to FDDI, BuST improves (as FDDI-M) the bandwidth available for real-time messages and halves the bandwidth lost due to the protocol overhead. Moreover, $\mathrm{BuST}$ is able to deliver non real-time traffic also in those cases in which FDDI-M fails, as shown in Section 3.2.1.

The overhead consisting in the bandwidth lost when an asynchronous message transmission is interrupted can be easily included into $\tau$. The same observation can be done for the overhead due to the fact that an in-progress transmission is not interrupted until its completion. This also holds for FDDI and FDDI-M.

As a final remark, the implementation of BuST only requires one timer, instead of the two timers needed by FDDI and FDDI-M. This can be useful when BuST is adopted in small embedded systems, where resources (i.e., hardware timers) are scarce.

\begin{tabular}{|l|l|}
\hline Allocation scheme & Assignment rule \\
\hline Proportional (PA) & $H_{i}=U_{i}(T T R T-\tau)$ \\
Normalized Proportional (NPA) & $H_{i}=\frac{U_{i}}{U}(T T R T-\tau)$ \\
\hline
\end{tabular}

\section{Table 1. The Synchronous Budget Alloca- tion schemes considered in this paper.}

\subsubsection{Token regeneration}

A typical problem in timed-token networks is the token loss. In case of loss, the token must be regenerated to let the communication to continue.

For FDDI networks, when the counter $L_{c}$ at node $i$ becomes equal to 2 , the node assumes that the token is lost and the ring recovery process starts [10].

If $\Delta$ is the interval between two consecutive visits of the token at the same node, for BuST it holds

$$
\Delta \leq \sum_{i=1}^{n} H_{i}+\tau \leq T T R T .
$$

Using BuST, each node $i$ has a timer $T H R T_{i}$ which is reset $\left(T H R T_{i}=0\right)$ every time the node gets the token. If


the node assumes that the token is lost and the recovery process is started.

In the rest of the paper, all formal results are derived under the assumption that the token is not lost and the protocol operates in the absence of errors.

\section{SBA schemes and time properties}

The real-time guarantee of the stream set highly depends on the SBA scheme adopted for budgets assignment given the stream set parameters. Table 1 shows the SBA schemes considered in this paper. Such schemes have 
been extensively studied for FDDI and FDDI-M protocols, thus the time properties derived for BuST can be compared with the results available in the literature.

Looking at the assignment rules reported in Table 1, it can be observed that PA is based on local information, i.e., the budget $H_{i}$ referred to the $i$-th stream is calculated using parameters of $S_{i}$ only, while NPA uses global information.

Lemmas 1, 2, 3 calculate the bound on the maximum transmission time for real-time messages while using, respectively, BuST, FDDI-M and FDDI. As explicitly declared in each lemma, the results are valid for all the considered SBA schemes.

Lemma 1 Under the BuST protocol, for all $S B A$ schemes, if $T_{i} \geq T T R T, i=1, \ldots, n$, it holds

$$
\forall i, j: s_{i, j} \leq t_{i, j}+\left\lceil\frac{C_{i}}{H_{i}}\right\rceil\left(\sum_{r=1}^{n} H_{r}+\tau\right) .
$$

Proof. If $D_{i} \leq T_{i}$, then when the $j$-th message of the stream $S_{i}$ is ready at time $t_{i, j}$, it is the only one in the stream transmission queue. In the worst case, the node is using the portion $\delta$ of the budget $H_{i}$ left by message $(j-1)$ to transmit non real-time traffic. In this case, message $j$ cannot preempt the transmission, and in the worst case has to wait for $\delta+\sum_{r=1, r \neq i}^{n} H_{r}+\tau$ units of time before the node can start transmitting it. It follows that, in the worst case, the time to deliver a portion $H_{i}$ of the message is $\delta+\sum_{r=1, r \neq i}^{n} H_{r}+\tau+H_{i}=\sum_{r=1}^{n} H_{r}+\tau+\delta$. To transmit an entire message of length $C_{i}$, a node needs $\left\lceil\frac{C_{i}}{H_{i}}\right\rceil$ token visits, and $\delta=\left\lceil\frac{C_{i}}{H_{i}}\right\rceil H_{i}-C_{i}$.

Two cases are possible:

1. If $\frac{C_{i}}{H_{i}} \in \mathbb{N}$, then $\left\lceil\frac{C_{i}}{H_{i}}\right\rceil=\frac{C_{i}}{H_{i}}$ and $\delta=0$. Hence, the message is completely delivered after $\left\lceil\frac{C_{i}}{H_{i}}\right\rceil \sum_{r=1}^{n}\left(H_{r}+\tau\right)$ units of time, and the lemma follows.

2. If $\frac{C_{i}}{H_{i}} \notin \mathbb{N}$, then at time $t_{i, j}+\left\lfloor\frac{C_{i}}{H_{i}}\right\rfloor\left(\sum_{r=1}^{n} H_{r}+\tau\right)+\delta$, the budget $H_{i}$ is consumed and the node stops transmitting the message. The remaining portion $C_{i}-$ $\left\lfloor\frac{C_{i}}{H_{i}}\right\rfloor H_{i}$ can be delivered after $\sum_{r=1}^{n} H_{r}+\tau-H_{i}$ units of time. In fact, the transmission of the $j$-th message can be completed because $C_{i}-\left\lfloor\frac{C_{i}}{H_{i}}\right\rfloor H_{i}<$ $C_{i}-\left(\frac{C_{i}}{H_{i}}-1\right) H_{i}=H_{i}$.

Now, since $\frac{C_{i}}{H_{i}} \neq\left\lceil\frac{C_{i}}{H_{i}}\right\rceil$, then $\left\lceil\frac{C_{i}}{H_{i}}\right\rceil=\left\lfloor\frac{C_{i}}{H_{i}}\right\rfloor+1$. Hence, $\delta=\left\lceil\frac{C_{i}}{H_{i}}\right\rceil H_{i}-C_{i}=\left\lfloor\frac{C_{i}}{H_{i}}\right\rfloor H_{i}+H_{i}-C_{i}$, and the maximum transmission time for the $j$-th message in stream $S_{i}$ becomes:

$$
\begin{aligned}
s_{i, j} \leq & t_{i, j}+\left\lfloor\frac{C_{i}}{H_{i}}\right\rfloor\left(\sum_{r=1}^{n} H_{r}+\tau\right)+\delta+ \\
& +\sum_{r=1}^{n} H_{r}+\tau-H_{i}+C_{i}-\left\lfloor\frac{C_{i}}{H_{i}}\right\rfloor H_{i} \\
s_{i, j} \leq & t_{i, j}+\left(\left\lfloor\frac{C_{i}}{H_{i}}\right\rfloor+1\right)\left(\sum_{r=1}^{n} H_{r}+\tau\right)+\delta-\delta \\
s_{i, j} \leq & t_{i, j}+\left\lceil\frac{C_{i}}{H_{i}}\right\rfloor\left(\sum_{r=1}^{n} H_{r}+\tau\right)
\end{aligned}
$$

Lemma 2 Under the FDDI-M protocol, for all $S B A$ schemes, if $T_{i} \geq T T R T, i=1, \ldots, n$, it holds

$$
\forall i, j: s_{i, j} \leq t_{i, j}+\left\lceil\frac{C_{i}}{H_{i}}\right\rceil \operatorname{TTRT}+C_{i}-\left\lceil\frac{C_{i}}{H_{i}}\right\rceil H_{i} .
$$

Proof. Being $\forall i: D_{i} \leq T_{i}$, when a message becomes ready to be sent for stream $S_{i}$, it is the only one in the transmission queue $S_{i}$. In the worst case, a message arrives just after the token left the node, therefore it has to wait for at most TTRT $-H_{i}$ units of time. When the token arrives, the node transmits the message for $H_{i}$ time units, hence a portion of the message equal to $H_{i}$ is transmitted at most every TTRT time units. Node $i$ needs $\left\lceil\frac{C_{i}}{H_{i}}\right\rceil$ token visits to transmit a message. Let distinguish two cases:

1. If $\frac{C_{i}}{H_{i}} \in \mathbb{N}$, then $s_{i, j} \leq t_{i, j}+\left\lceil\frac{C_{i}}{H_{i}}\right\rceil T T R T=t_{i, j}+$ $\frac{C_{i}}{H_{i}} T T R T$ and the lemma follows.

2. If $\frac{C_{i}}{H_{i}} \notin \mathbb{N}$, then after $\left\lfloor\frac{C_{i}}{H_{i}}\right\rfloor T T R T$ the token leaves the stream and, in the worst case, comes back after TTRT $-H_{i}$ units of time; at this point, the message delivery needs $C_{i}-\left\lfloor\frac{C_{i}}{H_{i}}\right\rfloor H_{i}<H_{i}$ units of time to complete. It follows that $s_{i, j} \leq t_{i, j}+$ $\left\lfloor\frac{C_{i}}{H_{i}}\right\rfloor T T R T+\left(T T R T-H_{i}\right)+C_{i}-\left\lfloor\frac{\overline{C_{i}}}{H_{i}}\right\rfloor H_{i}=$ $t_{i, j}+\left(\left\lfloor\frac{C_{i}}{H_{i}}\right\rfloor+1\right) T T R T+C_{i}-\left(\left\lfloor\frac{C_{i}}{H_{i}}\right\rfloor+1\right) H_{i}=$ $t_{i, j}+\left\lceil\frac{C_{i}}{H_{i}}\right\rceil T T R T+C_{i}-\left\lceil\frac{C_{i}}{H_{i}}\right\rceil H_{i}$.

Lemma 3 Under the FDDI protocol, for all $S B A$ schemes, if $T_{i} \geq 2 T T R T, i=1, \ldots, n$, it holds

$\forall i, j: s_{i, j} \leq t_{i, j}+\left(\left\lceil\frac{C_{i}}{H_{i}}\right\rceil+1\right) T T R T+C_{i}-\left\lceil\frac{C_{i}}{H_{i}}\right\rceil H_{i}$.

Proof. Being $\forall i: D_{i} \leq T_{i}$, when a message arrives to stream $S_{i}$, it is the only one in the transmission queue. Assume that a time $t_{i}(0)$, a real-time message arrives at node $i$; let $t_{i}(k)$ be the time of token's $k$-th arrival after $t_{i}(0)$. Then, from [5]:

$$
t_{i}(k)-t_{i}(0)=k T T R T+T T R T-H_{i} .
$$

It follows that, in the worst case, the message arrives just after the token left the stream, and it has to wait for at most $2 T T R T-H_{i}$ to start transmitting its real-time traffic.

To deliver an entire message of length $C_{i}$, node $i$ needs at most $k=\left\lceil\frac{C_{i}}{H_{i}}\right\rceil$ token visits. At time $t(k)$, the token comes back to node $i$; to complete the transmission of the message, the node transmits for $\delta_{i}=H_{i}-\left(\left\lceil\frac{C_{i}}{H_{i}}\right\rceil H_{i}-\right.$ $\left.C_{i}\right) \leq H_{i}$ time units. It follows that, from Equation 1:

$$
\begin{aligned}
& s_{i, j} \leq t_{i, j}+\left(\left\lceil\frac{C_{i}}{H_{i}}\right\rceil+1\right) T T R T-H_{i}+\delta_{i} \\
& s_{i, j} \leq t_{i, j}+\left(\left\lceil\frac{C_{i}}{H_{i}}\right\rceil+1\right) T T R T+C_{i}-\left\lceil\frac{C_{i}}{H_{i}}\right\rceil H_{i} .
\end{aligned}
$$




\section{SBA schemes analysis}

The results reported in Section 4 allow proving the properties for the allocation schemes applied to the BuST protocol. Moreover, they can be used to derive new properties for the same schemes under FDDI and FDDI-M. When not differently indicated, the following results refer to the BuST protocol.

Notice that, even though we assume $D_{i}=T_{i}$ for all the streams, since $U_{i}=\frac{C_{i}}{\min \left(D_{i}, T_{i}\right)}$, the same results can be derived for the case where $D_{i}<T_{i}$ by simply substituting $D_{i}$ to $T_{i}$.

To make the treatment clearer, let $\beta_{i}=\frac{T_{i}}{T T R T}$ and $\alpha=$ $\frac{\tau}{T T R T}$. Parameter $\alpha$ represents the bandwidth loss due to the overhead.

Due to the lack of the space we only show the analysis for the PA scheme. Readers interested in the analysis of other SBA schemes, as NPA, can refer to [7].

\subsection{Proportional Allocation}

Theorem 1 provides the WCAU for the Proportional Allocation scheme.

Theorem 1 The WCAU factor of the PA scheme, in which $H_{i}=U_{i}(T T R T-\tau)$, is equal to $\frac{1-3 \alpha}{2(1-\alpha)}$, where $\alpha=$ $\frac{\tau}{T T R T}$.

Proof. We show that the Protocol Constraint is satisfied for any TTRT and for $U \leq 1$ :

$$
\begin{aligned}
& \frac{1}{\operatorname{TTRT}} \sum_{i=1}^{n} H_{i}=\frac{1}{\operatorname{TTRT}} \sum_{i=1}^{n} U_{i}(\operatorname{TTRT}-\tau)= \\
& \sum_{i=1}^{n} U_{i}(1-\alpha)=U(1-\alpha) \leq 1-\alpha
\end{aligned}
$$

For the Deadline Constraint, from Lemma 1 it holds:

$$
\begin{gathered}
s_{i, j} \leq t_{i, j}+\left\lceil\frac{C_{i}}{H_{i}}\right\rceil\left(\sum_{r=1}^{n} H_{r}+\tau\right)= \\
t_{i, j}+\left\lceil\frac{\beta_{i}}{1-\alpha}\right\rceil\left(\sum_{r=1}^{n}\left(U_{r}(T T R T-\tau)\right)+\tau\right) \leq t_{i, j}+T_{i}
\end{gathered}
$$

Then to satisfy the deadline for any message in the stream $S_{i}$, the following inequality must be satisfied:

$$
\left\lceil\frac{\beta_{i}}{1-\alpha}\right\rceil\left(\sum_{r=1}^{n}\left(U_{r}(T T R T-\tau)\right)+\tau\right) \leq T_{i}
$$

We can write the first term as follows:

$$
\begin{aligned}
& \left\lceil\frac{\beta_{i}}{1-\alpha}\right\rceil\left(\sum_{r=1}^{n}\left(U_{r}(T T R T-\tau)\right)+\tau\right)= \\
& \left\lceil\frac{\beta_{i}}{1-\alpha}\right\rceil(U(T T R T-\tau)+\tau)= \\
& \left\lceil\frac{\beta_{i}}{1-\alpha}\right\rceil(T T R T-\tau)\left(U+\frac{\alpha}{1-\alpha}\right)
\end{aligned}
$$

The inequality can be rewritten as follows:

$$
\begin{aligned}
& \left\lceil\frac{\beta_{i}}{1-\alpha}\right\rceil(\operatorname{TTRT}-\tau)\left(U+\frac{\alpha}{1-\alpha}\right) \leq T_{i} \\
& \left\lceil\frac{\beta_{i}}{1-\alpha}\right\rceil\left(U+\frac{\alpha}{1-\alpha}\right) \leq \frac{\beta_{i}}{1-\alpha} \\
& U \leq \frac{\frac{\beta_{i}}{1-\alpha}}{\left\lceil\frac{\beta_{i}}{1-\alpha}\right]}-\frac{\alpha}{1-\alpha}
\end{aligned}
$$

Being $\beta_{i} \geq 1$ and $\alpha \geq 0$, then $\frac{\beta_{i}}{1-\alpha} \geq 1$. It is simple to view that (see [7]) $\frac{1}{2} \leq \frac{\frac{\beta_{i}}{1-\alpha}}{\left\lceil\frac{\beta_{i}}{1-\alpha}\right\rceil}$, hence it follow that, if

$$
U \leq \frac{1}{2}-\frac{\alpha}{1-\alpha}=\frac{1-3 \alpha}{2(1-\alpha)}
$$

the Deadline Constraint is satisfied.

Theorem 1 considers the worst case for the streams utilization $U$ that guarantees the stream set schedulability. The BuST initialization rule states that the TTRT is chosen equal to $\min _{i}\left(T_{i}\right)$. However, in some cases it is possible to choose $T T R T<\min _{i}\left(T_{i}\right)$ such that the upper bound for the utilization can be greater than WCAU.

The following corollaries extend the result provided by Theorem 1.

Corollary 1 For $i=1, \ldots, n$, and $T T R T \leq T_{i}$, if

$$
U \leq \frac{\min _{i}\left(\beta_{i}\right)}{(1-\alpha)\left\lceil\frac{\min _{i}\left(\beta_{i}\right)}{1-\alpha}\right\rceil}-\frac{\alpha}{1-\alpha}
$$

then the stream set $M=\left\{S_{1}, \ldots, S_{n}\right\}$ is schedulable under the PA scheme.

Proof. The proof is very similar to that of Theorem 1 . See [7] for further details. $\square$

It is worth observing that, if $\frac{T_{i}}{T T R T(1-\alpha)} \in \mathbb{N}$, then WCAU can be greater than the value given in Theorem 1 . Corollary 2 shows how to properly set TTRT to satisfy that condition.

Corollary 2 If TTRT $=G C D_{i}\left(T_{i}\right)+\tau$, the WCAU factor of the PA scheme is equal to $\frac{1-2 \alpha}{1-\alpha}$ for BuST, and equal to $1-\alpha$ for FDDI-M.

Proof. See [7].

Notice that, if TTRT $=G C D_{i}\left(T_{i}\right)+\tau$, then $T T R T \geq 1+\tau$. Therefore, by setting TTRT as stated in Corollary 2 and substituting it in $\frac{1-2 \alpha}{1-\alpha}$, the worst-case value of WCAU results to be $1-\tau$. This means that WCAU can be much smaller than the value given in Theorem 1, depending on the value of $\tau$. However, there are several cases in which, using the last two corollaries, it is possible to guarantee a bandwidth greater than that provided by Theorem 1. For instance, let us consider three message streams having period $7 \mathrm{~ms}, 15 \mathrm{~ms}$ and $13 \mathrm{~ms}$, respectively; it follows that $\operatorname{GCD}(7,15,13)=1$ $m s$. Suppose $\tau=0.2 \mathrm{~ms}$, hence if the channel utilization $U \leq 1-\tau=0.8$ then the stream set is schedulable. The same example can be used to show that, if we choose for 
instance $T T R T=\min _{i}\left(T_{i}\right) / 2=3.5 \mathrm{~ms}$, then by Corollary 1 , if the channel utilization $U \leq 0.65$, the stream set is schedulable. Note that, if $T T \bar{R} T=\min _{i}\left(T_{i}\right)$, as stated in the standard rules of BuST, then the WCAU is $\frac{1-3 \alpha}{2(1-\alpha)}=0.49$, that is, we can guarantee less bandwidth than in the previous cases.

For FDDI [5] and FDDI-M [4], the WCAU for the PA scheme can asymptotically approach $0 \%$. However, by setting TTRT as suggested in Corollary 2, we can get a non-null WCAU also for FDDI-M.

\subsection{Discussion of theoretic results}

For the PA scheme, the BuST protocol has a WCAU equal to $\frac{1-3 \alpha}{2(1-\alpha)}$, whereas with FDDI and FDDI-M no stream set can be guaranteed. For the PA scheme, under BuST, there exists an upper bound on the utilization $U$ that depends on $\alpha$ and on the minimum ratio $\beta_{i}$ (refer to Corollary 1).

Corollary 2 state that, for PA, the WCAU for BuST and FDDI-M can be improved by properly setting TTRT. In particular, the WCAU can get close to 1 when $\alpha$ is negligible. However, TTRT may become very small, so that $\alpha=\frac{\tau}{T T R T}$ becomes big, resulting in a small available bandwidth, it is depends on the value of $\tau$.

\subsection{Non real-time service}

So far, real-time stream services have been analyzed. This section briefly describes the non real-time service of the BuST protocol and its improvements with respect to FDDI-M.

To analyze a worst-case scenario for the non real-time service, we assume that each node receiving the token has always non real-time traffic to deliver. In this case, the total channel utilization of the network, including the realtime and the non real-time traffic, is equal to $1-\alpha$. The following theorem provides the minimum bandwidth that a node $i$ can exploit to deliver non real-time traffic.

Theorem 2 Under the BuST protocol, a node $i$ can guarantee for the non real-time traffic a minimum bandwidth $U_{i}^{n r t}$, which depends on the SBA scheme adopted. In particular:

$$
\begin{aligned}
& \text { - with } P A, U_{i}^{n r t}=U_{i}\left(\frac{1}{U+\frac{\alpha}{1-\alpha}}-1\right) \\
& \text { - with NPA, } U_{i}^{n r t}=U_{i}\left(\frac{1-\alpha}{U}-1\right)
\end{aligned}
$$

Proof. To prove the theorem is sufficient to note that the maximum bandwidth available to node $i$ to deliver both real-time and non real-time traffic is $U_{i}^{\text {tot }}=\frac{H_{i}}{\sum_{j=1}^{n} H_{j}+\tau}$. It follows that, being $U_{i}$ the utilization of the real-time messages at node $i$, the minimum bandwidth available for the non real-time messages at the same node results to be $U_{i}^{n r t}=U_{i}^{t o t}-U_{i}$. For instance, let us calculate $U_{i}^{n r t}$ with the NPA scheme, where $H_{i}=\frac{U_{i}}{U}(T T R T-\tau)$ :

$$
\begin{aligned}
& U_{i}^{t o t}=\frac{H_{i}}{\sum_{j=1}^{n} H_{j}+\tau}=\frac{U_{i}(1-\alpha)}{U} \\
& U_{i}^{n r t}=U_{i}^{t o t}-U_{i}=\frac{U_{i}(1-\alpha)}{U}-U_{i}= \\
& U_{i}\left(\frac{1-\alpha}{U}-1\right) .
\end{aligned}
$$

The result for the PA scheme can be derived in a similar way. $\square$

We have shown that, under the BuST protocol, the non real-time traffic at each node has a minimum bandwidth guaranteed. In addition, it is worth observing that, when not all nodes of the network have to send non real-time traffic, during the round trip of the token, the value of $U_{i}^{n r t}$ can increase.

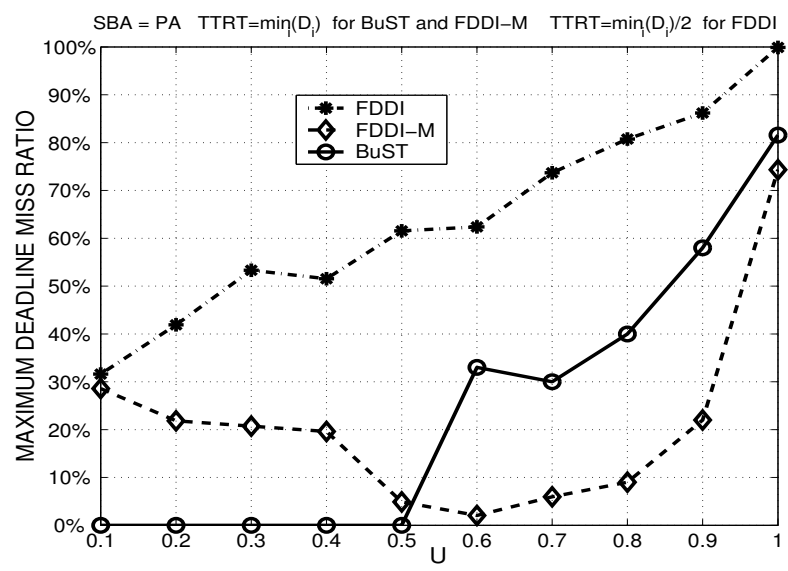

Figure 1. Maximum Deadline Miss Ratio for the PA scheme.

\section{Simulation results}

In this section the performance of BuST, FDDI and FDDI-M is compared by simulation. The reported results refer to a network consisting of 10 nodes. Each node has a periodic stream with a relative deadline ranging from $10 \mathrm{msec}$ to $100 \mathrm{msec}$, and an infinite amount of non realtime traffic, i.e. every time a node receives the token it has some non real-time traffic to deliver. Considering a protocol overhead for each node equal to $2 \mu \mathrm{sec}$, it follows that $\tau=20 \mu \mathrm{sec}$. We assigned the node budgets using the Proportional Allocation scheme analyzed in the previous section. We measured the Maximum Deadline Miss Ratio varying the total channel utilization $U$ from 0.1 to 1.0 , with a step of 0.1 . For each value of $U$ we simulated the protocols with 500 runs, taking the Maximum value of the Deadline Miss Ratio. For each run we have generated a different stream set.

Figure 1 reports the Maximum Deadline Miss Ratio. As expected from the analysis in Section 5.1, as long as $U \leq \frac{1-3 \alpha}{2(1-\alpha)} \simeq 0.5$, BuST has no deadline miss, while FDDI and FDDI-M present a non-null deadline miss ratio. For $U>0.5 \mathrm{BuST}$ starts experiencing deadline misses, and FDDI-M has the lowest deadline miss ratio among the three protocols.

Figure 2 shows the results obtained when, as stated in Corollary 2, TTRT $=G C D_{i}\left(D_{i}\right)+\tau$. In this case, as long as $U \leq 0.9$, both BuST and FDDI-M do not experience deadline misses. In fact, being $\alpha$ very small, the value of $U^{*}$ for both BuST and FDDI-M is close to 1 (see Corollary 2).

Notice that for FDDI we have deadline misses for all values of $U$.

We also simulated the behavior of the protocols when there is only real-time traffic in the network, that is, when the nodes has no non real-time traffic to deliver. In this case, FDDI, FDDI-M and BuST operate in the same way, since they are in practice the same protocol. In this case, in fact, the maximum token rotation time does not exceed $T T R T$ even for FDDI. Therefore, TTRT can be set to 
$\min _{i}\left(D_{i}\right)$ for the three protocols. The results are reported in Figure 3. As long as $U$ is not greater than 0.6, there are not deadline misses. With $U=0.7$ we have a very small Maximum Deadline Miss Ratio, which is equal to $0.09 \%$; note that, in the graph this value is not appreciable because is too small.

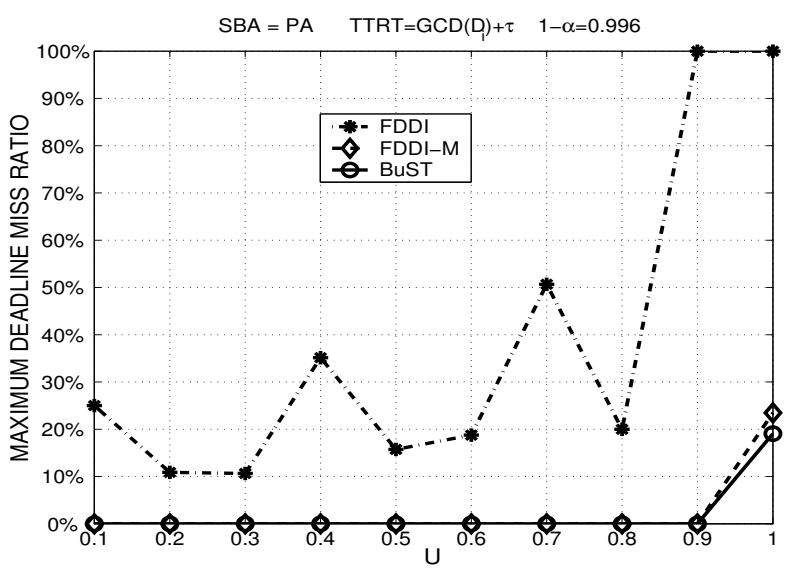

Figure 2. Maximum Deadline Miss Ratio with $T T R T=G C D_{i}\left(D_{i}\right)+\tau$.

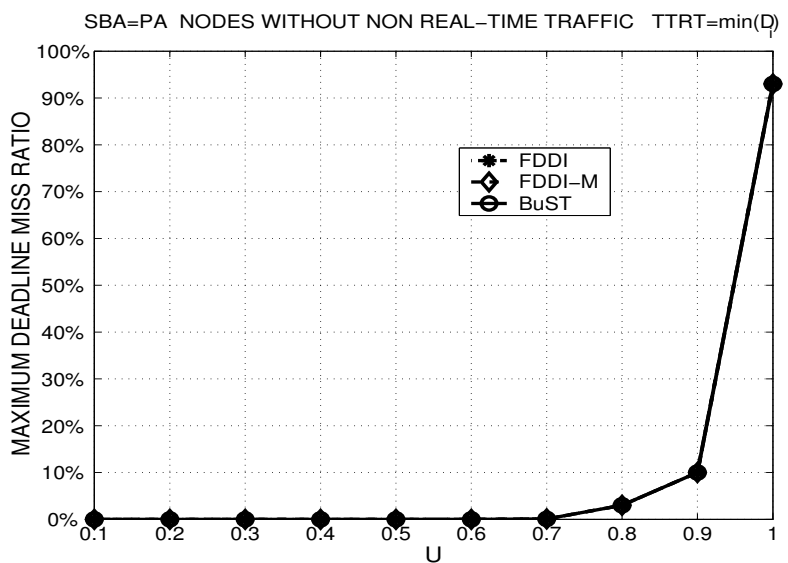

Figure 3. Maximum Deadline Miss Ratio without non real-time traffic.

\section{Conclusions}

This paper described BuST, a new token passing protocol for guaranteeing timing constraints of periodic message streams and minimum throughput for non real-time traffic. A budget allocation scheme available in the literature has been analyzed, to show the benefits of the proposed communication policy. Theoretical results show that BuST has equal or better performance with respect to the previous approaches. Furthermore, we showed how to set the value of the TTRT to improve the bandwidth guaranteed for real-time streams under the $P A$ scheme. We simulated the discussed protocols to confirm the theoretic results, and to evaluate their behavior under different conditions.

As a future work, we plan to perform additional simulations of BuST, FDDI and FDDI-M to compare their performance with different non real-time traffic models. Moreover, we plan to compare BuST with other token passing protocols as, for instance, TDMA/SS [2]. We finally plan to extend the analysis to other SBA schemes proposed in the literature for timed-token protocols and further investigate token loss problems related to the schedulability of real-time streams.

\section{References}

[1] G. Agrawal, B. Chen, W. Zhao, and S. Davari. Guaranteeing Synchronous Message Deadlines with the Timed Token Medium Access Control Protocol. IEEE Trans. on Computer, 43(3):327-339, March 1994.

[2] B. Andersson, E. Tovar, and N. Pereira. Analysing TDMA with Slot Skipping. In Proceedings of the 26th IEEE RealTime Systems Symposium, Dec 2005.

[3] G. Bussiere, J. Oblinger, and V. F. Wolfe. Real-time considerations in submarine target motion analysis. In Proc. of Proceedings of the IEEE Workshop on Real-Time Applications, May 1993.

[4] E. Chan, D. Chen, J. Cao, and C.-H. Lee. Time Properties of the FFDI-M Medium Access Protocol. The Computer Journal, 82(1):96-102, Jan. 1999.

[5] B. Chen and W. Zhao. Properties of the Timed Token Protocol, 92-038. Technical report, Dept. of Computer Science, Texas A\&M University College Station, Oct. 1992.

[6] G. Franchino, G. C. Buttazzo, and T. Facchinetti. A Distributed Architecture For Mobile Robots Coordination. In Proc. of the 10th IEEE International Conference on Emerging Technologies and Factory Automation (ETFA 2005), Sept. 2005.

[7] G. Franchino, G. C. Buttazzo, and T. Facchinetti. A New Token Passing Protocol for Real-Time Communication. Technical report, University of Pavia, http://robot.unipv.it/media/publications/UNIPVRoboLab-Franchino-TR01-07.pdf, 2007.

[8] R. Grow. A Timed Token Protocol for Local Area Networks. In Proc. Electro'82, Token Access Protocols, Paper 17/3, May 1982.

[9] M. Hamdaoui and P. Ramanathan. Selection of Timed Token Parameters to Guarantee Message Deadlines. IEEE/ACM Trans.on Networking, 3(3):340-351, 1995.

[10] M. Johnson. Reliability Mechanisms of the FDDI High Bandwidth Token Ring Protocol. Comput. Net. ISDN Syst., 2(1):121-131, 1986.

[11] R. J. Kochanski and J. L. Paige. SAFENET: The Standard and Its Application. IEEE LCS, 2(1):46-51, 1991.

[12] N. Malcolm and W. Zhao. The timed-token protocol for real-time communications. Computer, 27(1):35-41, Jan. 1994.

[13] J. K.-Y. Ng. MPEG transmission schemes for a timed token medium access control network. SIGCOMM Comput. Commun. Rev., 29(1):66-80, 1999.

[14] K. Sevcik and M. Johnson. Cycle Time Properties of the FDDI Token Ring Protocol. IEEE Trans. on Software Eng., 13(3):376-385, March 1987.

[15] K. G. Shin and Q. Zheng. FDDI-M: A Scheme to Double FDDI's Ability of Supporting Synchronous Traffic. IEEE Trans. on Parallel and Distrib. Syst., 6(11):1125-1131, Nov. 1995

[16] E. Tovar and F. Vasques. Cycle time properties of the PROFIBUS timed-token protocol. Computer Communications, Elsevier Science, 22(13):1206-1216, 1999.

[17] S. Zhang, A. Burns, J. Chen, and E. Lee. Hard Real-Time Communication with the Timed Token Protocol: Current State and Challenging Problems. Real-Time Systems, 27(3):271-295, Sept. 2004.

[18] S. Zhang, A. Burns, and A. Wellings. An Efficient and Practical Local Synchronous Bandwidth Allocation Scheme for The Timed-Token MAC Protocol. In Proc. of the IEEE Infocom'96, pages 920-927, 1996. 